\title{
CORRECTION
}

\section{Correction to: Genome-wide mapping of adult plant stripe rust resistance in wheat cultivar Toni}

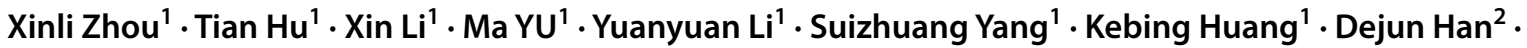 \\ Zhensheng Kang ${ }^{2}$
}

Published online: 30 May 2019

๑) Springer-Verlag GmbH Germany, part of Springer Nature 2019

\section{Correction to: Theoretical and Applied Genetics https://doi.org/10.1007/s00122-019-03308-1}

Unfortunately, Figs. 2 and 3 were interchanged in the results section. The figures should swap positions, whereas the legends should stay in the given order.

The correct Figs. 2 and 3 are given below:

1 Wheat Research Institute, School of Life Sciences and Engineering, Southwest University of Science and Technology, Mianyang 621010, Sichuan,

People's Republic of China

2 State Key Laboratory of Crop Stress Biology in Arid Areas and College of Plant Protection, Northwest A\&F University, Yangling 712100, Shaanxi, China 


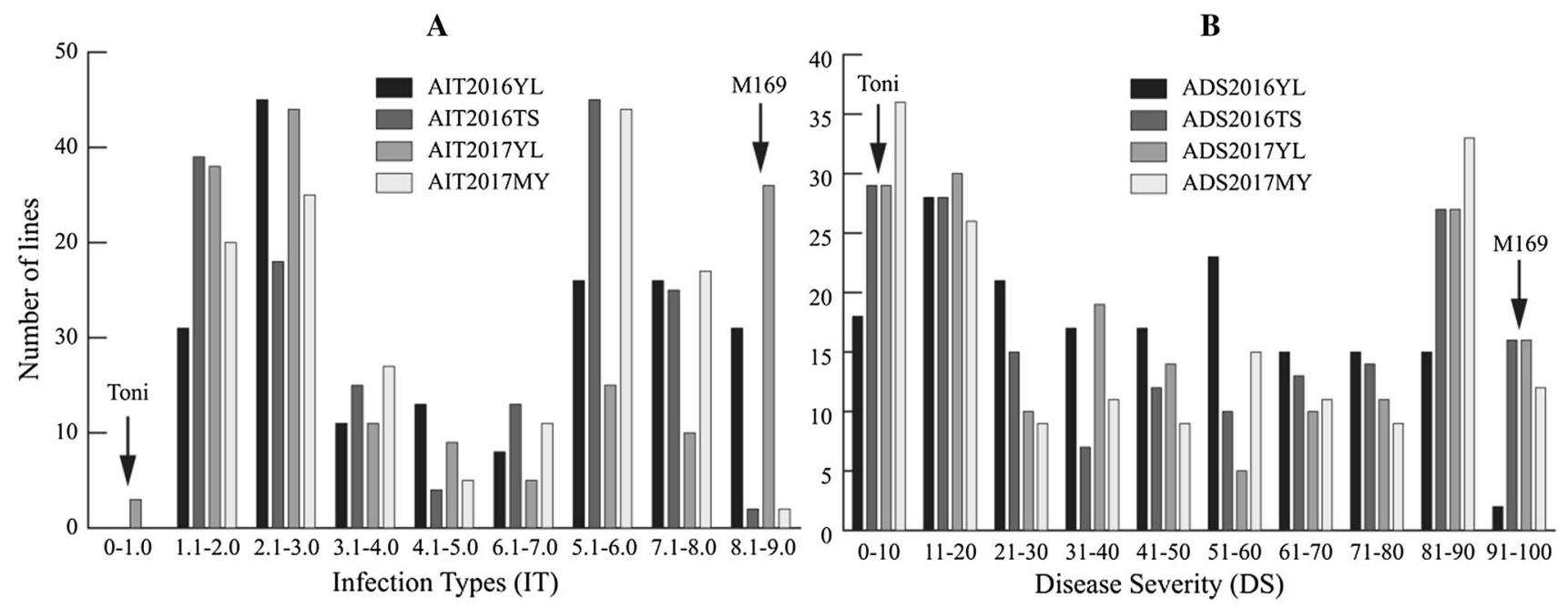

Fig. 2 Frequency distributions of mean infection types (IT) (A) and disease severities (DS) for $171 \mathrm{~F}_{7}$ RILs from cross Mingxian169 $\times$ Toni grown at Yangling and Tianshui in 2016-2017. Arrows indicate the values of the parental lines

Fig. 3 Circos diagram showing LOD values on genetic maps for 21 wheat chromosomes. Track a chromosomes and genetic scale (cM). Track b stripe rust resistance QTL detected by ICIM in the M169/Toni RIL population across four environments based on mean infection type. Track c stripe rust resistance QTL detected by ICIM in the M169/ Toni RIL population across four environments based on mean disease severity (MDS) (track c)

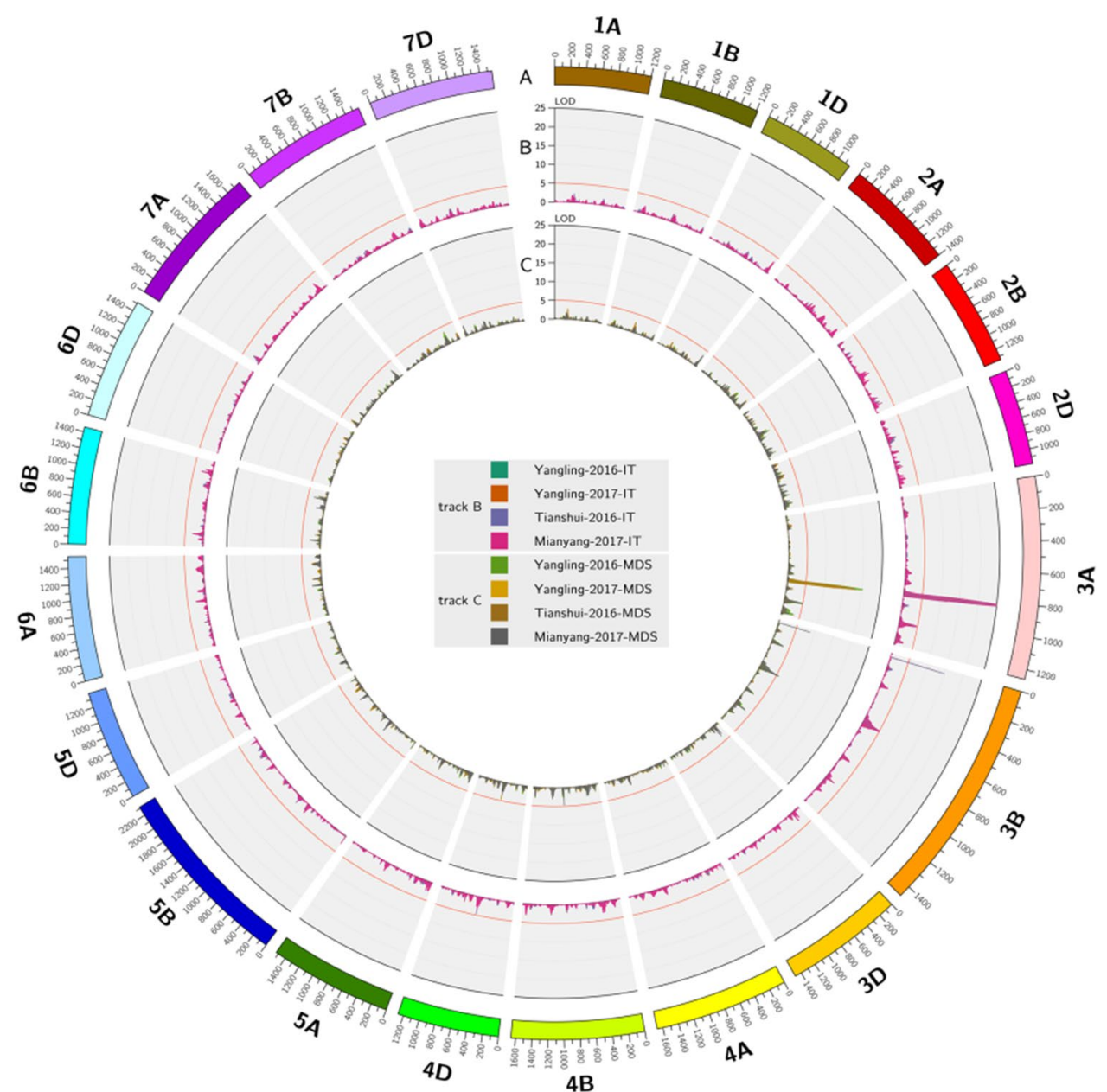

Publisher's Note Springer Nature remains neutral with regard to jurisdictional claims in published maps and institutional affiliations. 